\title{
Developments of Flame Propagation in Micro and Mesoscale Channels
}

\author{
Vihar D.Nandan ${ }^{\# 1}$, Narendra Deore ${ }^{* 2}$, \\ \#Department of Mechanical Engineering, PCCOE, Pune University, Pune, INDIA \\ \# Department of Mechanical Engineering, PCCOE, Pune University, Pune, INDIA
}

\begin{abstract}
Flame propagation through channels with wall interaction and different types of flames are studied in the present work. Study includes propagation velocity of flame in accordance with burning velocity. The mean velocity and concentration gradient of fuel will be controlled by various types of burners. The propagation and extinction in a channel is studied to understand the effect of heat transfer between flame and wall on burning rate. The behaviour of flame and flame structure on basis of variations in local mixture composition will be studied which extends to physical and chemical parameters of a particular flame configuration. While studying propagation of flame in channels it is needed to study spinning behaviour of flames, asymmetry of flames, stretched flames and unstable flames. Various structures of flames make it unstable and directly affect on combustion. Mainly experimental study of combustion process in accordance with channel dimensions, flow rate, equivalence ratio and flame speed needs to be focused. Special attention is devoted to symmetry of flames and flame wall coupling.
\end{abstract}

Keywords: - Flame wall coupling, mesoscale channel, stretched flames, spinning flames

\section{A .Developments and Research}

\section{INTRODUCTION}

Recent Development in micro mechanical, portable, communicational, sensing, chemical biomedical and aerospace propulsion devices have opened up broad new area of active research into micro and Mesoscale channels. Today these devices heavily rely on batteries. The energy density of existing batteries is very low, even when it comes to advanced lithium ion, its only about $0.20 \mathrm{Kwh} / \mathrm{kg}$. This energy backup support only for few hours e.g. application like computer notebook. The micro-combustor, burning fossil fuel, are projected to replace the batteries for such applications.

Many investigations have been reported in literature on flame propagation in micro channels. Main focus was on parameters like flame speed, quenching of chemical reactions, radical quenching, channel width, flow velocity, heat loss and flame structure interaction. Working in silicon channel researcher came with result that flame does not propagate at constant speed through channel for rich mixture and it almost comes to freeze, then accelerates and exits channel at maximum velocity. This phenomenon very well studied as repetitive ignition and extinction of flame. Heat transfer from flame to wall be the cause of reduction in temperature of flame and also it leads to extinction of flame [1]. The phenomenon as stretch of flame widely depends on channel dimensions and inflow velocity. Effect of channel width causes some significant changes like at larger width of channel, flame is negatively stretched and by decreasing the dimensions causes the effect of flame extinction and also possibility of transition of flame branch [2]. Experimental and theoretical results showed that the dependence of flame speed on channel width and equivalence ratio is nonlinear and is strongly affected by flow rate, two distinct flame regime also be observed in accordance with changes in dimensions [3]. Wall thermal properties have dramatic effects on flame propagation and its burning rate in channels.

Experimental investigation for methane and propane air premixed flames in Mesoscale channels showed that for fixed equivalence ratio, there was flow rate above which flame starts spinning and spin frequency was approximately proportional to flame speed. It was also be observed that spinning flame only occurred after transition from fast regime to slow regime. Having done with this it was observed that spin frequency and flame speed causes direct effect on each other [4]. While analysing flame in channels the tribranchial flames structure also be studied. The critical concentration gradient plays an important role on tirbranchial flames and its propagation velocity. The critical concentration gradient increased to larger value when the convective diffusion was enhanced [5]. Researcher did experimental work on flame propagation with methane-air in narrow channels and they came with conclusion that the flame speed increases and flammability limit shift towards fuel lean direction and it would either by increasing the preheat temperature or by decreasing the mixture flow rate or sometimes both[6]. The Lewis number which is also be the important parameter in channel flow and which is used to characterize fluid flow. The heat loss effect and Lewis number together for laminar premixed flames propagating in channels tells that at low Lewis number for fluid flow opposing the 
flow motion, increases in heat loss leads to the transition from inverted "Mushroom" to "Funnel-shaped" flame[7]. While working in radial microchannels for methane-air mixture the rotating pelton like flame structure were observed experimentally and the stable flames observed at radial location for stoichiometric and rich mixture over a wide range of operating conditions. It was observed that for lean methane-air mixture at equivalence ratio of 0.67 and channel gap of $1-2 \mathrm{~mm}$, distinct pelton like rotating flame structure were observed [8]. Again for same mixture with different air-fuel mixtures the spiral flames were observed. The transition from radial to spiral flame over a range of equivalence ratio, varying from 1.17 to 1.22 had been noticed experimentally [9].

The propagation of laminar pre-mixed flame front in diverging channels with various wall heat transfer conditions showed that an increase in flow velocity changes flame shape from "Mushroom" type to "Tulip" shaped. Flame position and flame transition also be depends on divergence angle for channel having divergence angle less than $10 \mathrm{deg}$ [10]. For stabilisation behaviour of flame in same diverging channels with the same premixed methane-air mixture it was noticed that to have complete combustion the stability of flame needs to maintain. The experimental investigations by Bhupendra Khandelwal et al. concluded as that the flame obtained for rich mixture had more stable nature as compared to lean and Flame stability limits were observed to vary in between $0.2 \mathrm{~m} / \mathrm{s}$ to $1.9 \mathrm{~m} / \mathrm{s}$ for range of mixture equivalence ratio and the divergent part plays significant role to have stable flame [11].The flame stretch also noticed for different types of flow rates and the negatively stretched flames were observed for moderate and rich mixtures and positively stretched for high flow rates. In every experimental study the downstream part of channel was heated to maintain positive wall temperature gradients along direction of fluid flow and which also helps to understand flame wall coupling and heat loss due to interaction [12]. The experimentation on flame stretch by Mohammad Akram et al. found that the stretch of flame can be avoided with high aspect ratio and preheated wall condition. The stretch free flames would also be utilised to have proper burning of flame and to analyse burning velocity of mixture at maximum temperature. Experimentally the planar flames also reported at the location of match between inflow velocity and burning velocity of mixture [25].

Flame structure interaction and conductive heat losses on flame propagation of pre-mixed flames in channel gives us that depending on channel width there are two types of possible modes of extinction, by excessive heat loss total flame extinction brought about in narrow channel and partial flame extinction near wall of wider channels [13]. Local flame extinction in flame front causes transition from stable circular flame to travelling flame and travelling to pelton like flame, also the local splitting of flame front can be a cause of transition from unstable circular flame to spiral flame, it was given that the asymmetric and unstable flow field can be a reason for local splitting of flame front and this work also be noticed by researcher experimentally [14]. Recently researcher came up with some new results of extinction characteristics and transition of same flames to ball like flames and which was examined in microgravity experiments and the two dimensional computations indicated that the temperature of ball like flame increased with the decrease of equivalence ratio in the near-limit condition when it approached to extinction[15]. To examine effect of Lewis number, wall temperature and tube diameter on flame propagation researcher worked with some analytical correlation which also account for heat loss and radical quenching[16].To have complete combustion and understanding of flame instabilities and transition researcher did experimental and numerical investigation of flame pattern formation in various microchannels. Experimental investigation of flame pattern formation in radial microchannels with a gap width of $1.75 \mathrm{~mm}$ showed that in addition to conventional stable flames some stationary flame patterns like single and double pelton like flames and the travelling flame were also observed. Flames rotated around centre of radial microchannel at a frequency of 25 to $55 \mathrm{~Hz}$. All the discussion based on the mixture equivalence ratio rang of 0.65 to 1.30 and inlet velocity range 1.5 to $3.0 \mathrm{~m} / \mathrm{s}$ [17]. It was also observed that rotating and oscillating flame patterns in a radial channel were observed only within certain range of gas flow rates beyond this range, at higher flow rates stationary flame mode took place. At smaller flow rates the instabilities leads to appearance of flame repetitive ignition/extinction in straight tube and formation of rotating flame pattern in radial channel. It was also found that rotating structures resembling spiral flame in radial channel and travelling waves in rectangular channels were found in some narrow range of gas flow rates [18].

Using various premixed fuel-air mixture in stepped tube micro combustion the formation of various steady and unsteady spinning flame propagation observed experimentally. Researcher found the propagating spinning flames for both, lean and rich mixture of methane, LPG, propane- air. The propagating mode found to range of flow rates and the mixture equivalence ratio due to thermal wall coupling. It was noticed that the spinning frequency of flames was around $150 \mathrm{~Hz}$ and which was almost twice the earlier work and this high frequency spinning flames leads to complete combustion with almost zero CO emissions. Spinning nature of flames can be a cause of combustion completeness [19].

The comprehensive study of flame pattern formation by Aiwu fan et al. tells that for with premixed methane air mixture and external heat by porous burner gives us various stable and unstable flame patterns. It was also concluded that the flame pattern is strong function of mixture equivalence ratio, inlet mixture velocity 
and channel width. The pelton like flame pattern either existed with low inlet mixture velocity or in case of low and high equivalence ratio. The stable circular flames not observed until the channel width was increased up to $2.00 \mathrm{~mm}$ [22]. The work by Gianmarco Pizza et al. on dynamics of premixed flames in mesoscale channels concluded that the various open and closed symmetry of flame were obtained with different inflow conditions and for increasing channel height flame dynamics become more complex and two new flame types are obtained, i.e. open symmetry and chaotic oscillating flame[23].

It is found that the quenching distance for heated silicon channels can be even below $0.7 \mathrm{~mm}$ and which has strong dependence on wall temperature. In steady flame the unburned gas mixture was preheated by hot wall and flame initialisation occurs. The quenching conditions between oscillating and steady flame is very narrow and vanishes for larger fuel flow rate [24].

\section{CONCLUSION}

Experimental investigations in micro channels showed that work needs to be focused on flame extinction, quenching of flame, repetitive extinction and ignition and asymmetry of flame and which occurs due to strong flame wall interaction. Various types of flames have been reported in literatures, flame in channels and spinning behaviour of flames, asymmetry of flames, stretched flames and unstable flames have also reported experimentally. The effect of flame wall heat transfer on propagation velocity has been studied. The asymmetry of wall also be the cause of incomplete combustion and which also leads to strong flame wall coupling. The heat loss caused by mixture at the inlet section can be avoided by heat recirculation method as well. To understand the propagation phenomenon the experimental study needs to focus on above dynamic instabilities of flame.

\section{REFERENCES}

[1] N.B.Brovig, D.C.Walther, A.C.Fernandes-Pello, D.Bjerketvedt, Experimental investigation of premixed flame propagation in silicon channels, Power MEMS Wokshop, (2005), pp.33-36.

[2] Yiguang Ju and $\mathrm{Bo} \mathrm{Xu}$, Effects of flow rate and kinetic quenching on the transition of flame regimes in mesoscale combustion.

[3] Bo Xu, Yiguang Ju, Theoretical and experimental studies on Mesoscale flame propagation and extinction, Proceedings Of The Combustion Institute, 30(2005) pp.2445-2453.

[4] Bo Xu, Yiguang Ju, Experimental Study Of Spinning Combustion In A Mesoscale Divergent Channel, Proceedings Of The Combustion Institute 31, 2007 pp.3285-3292.

[5] Nam Il Kim A, Jeong Il Seo B, Young Tae Guahk B, Hyun Dong Shin B, The Propagation Of Tribrachial Flames In A Confined Channel, Combustion And Flame 146, 2006 pp.168-179.

[6] C.Y.H. Chao A, K.S. Hui A, W. Kong A, P. Cheng B, J.H. Wang A, Analytical And Experimental Study Of Premixed Methane-Air Flame Propagation In Narrow Channels, International Journal Of Heat And Mass Transfer 50, 2007 pp.1302-1313.

[7] Subhadeep Chakraborty, Achintya Mukhopadhyay, Swarnendu Sen, Interaction of Lewis number and heat loss effects for a laminar premixed flame propagating in a channel, International Journal of Thermal Sciences 47 (2008)pp. 84-92.

[8] Sudarshan Kumar, Kaoru Maruta, S.Minaev, On the formation of multiple rotating Pelton-like flame structures in radial microchannels with lean methane-air mixtures, Proceedings of the Combustion Institute 31 (2007) pp.3261-3268

[9] Sudarshan Kumar, Kaoru Maruta, S. Minaev, and R. Fursenk, Appearance of target pattern and spiral flames in radial microchannels with CH4-air mixtures, physics of fluids 20, 024101, 2008, pp.1-11

[10] Sudarshan Kumar, Numerical Studies On Flame Stabilization Behaviour Of Premixed Methane-Air Mixtures In Diverging Mesoscale Channels, Combust. Sci. And Tech., 183: 2011, pp.779-801.

[11] Bhupendra Khandelwal, Sudarshan Kumar, Experimental investigation on flame stabilisation behaviour in a diverging microchannel with premixed methane air mixture.

[12] Mohammad Akram, Sudarshan Kumar, Experimental studies on dynamics of methane-air premixed flame in meso-scale diverging channels, Combustion and flame 158(2011)pp.915-924.

[13] J. Daou, M. Matalon, Influence Of Conductive Heat-Losses On The Propagation Of Premixed Flames In Channels, Combustion And Flame 128 (2002), pp.321-339.

[14] Aiwu Fan, Kaoru Maruta, Hisashi Nakamura, Wei Liu, Experimental investigation of flame pattern transition in radial micro-channel, Applied Thermal Engineering, volume 47, 5 December 2012, pp.111118.

[15] Koichi Takase, Xing Li, Hisashi Nakamura, Takuya Tezuka, Susumu Hasegawa, Masato Katsuta, Masao Kikuchi, Kaoru Maruta, Extinction characteristics of $\mathrm{CH} 4 / \mathrm{O} 2 / \mathrm{Xe} /$ radiative counter flow planar premixed flames and their transition to ball-like flames, Combustion and Flames 160 (2013) pp.1235-1241.

[16] Bin Bai, Zheng Chen, Huangwei Zhang, Shiyi Chen, Flame propagation in a tube with wall quenching of radicals, Combustion and Flame, (2013) pp.1-10. 
[17] Aiwu Fan, Sergey Minaev, Evgeniy Sereshchenko, Roman Fursenko, Sudarshan Kumar d, Wei Liu b, Kaoru Maruta, Experimental and numerical investigations of flame pattern formations in a radial microchannel, Proceedings of the Combustion Institute 32 (2009) pp.3059-3066

[18] Sergey Minaev, Roman Fursenko, Evgeniy Sereshchenko, Aiwu Fan, Sudarshan Kumar, Oscillating and rotating flame patterns in radial microchannels, Proceedings of the Combustion Institute 34 (2013) pp.3427-3434

[19] Anil A. Deshpande, Sudarshan Kumar, On the formation of spinning flames and combustion completeness for premixed fuel air mixtures in stepped tube microcombustors, Applied Thermal Engineering 51 (2013) pp. 91-101

[20] Yiguang Ju a, Kaoru Maruta, Microscale combustion: Technology development and fundamental research, Progress in Energy and Combustion Science xxx (2011) pp.1-47

[21] Vadim N. Kurdyumov a, Gianmarco Pizza b, Christos E. Frouzakis b, John Mantzaras c, Dynamics of premixed flames in a narrow channel with a step-wise wall temperature, Combustion and Flame 156 (2009) pp.2190-2200

[22] Aiwu Fan, Sergey Minaev, Sudarshan Kumar, Wei Liu, Kaoru Maruta, Regime diagrams and characteristics of flame patterns in radial microchannels with temperature gradients, Combustion and Flame 153 (2008) pp.479-489

[23] Gianmarco Pizza, Christos E. Frouzakis, John Mantzaras, Ananias G. Tomboulides, Konstantinos Boulouchos, Dynamics of premixed hydrogen/air flames in mesoscale channels, Combustion and Flame 155 (2008)pp. 2-20

[24] Yong Fan, Yuji Suzuki, Nobuhide Kasagi, Experimental study of micro-scale premixed flame in quartz channels, Proceedings of the Combustion Institute 32 (2009) pp.3083-3090

[25] Mohammad Akram, Sergey Minaev, and Sudarshan Kumar, Investigations on the formation of planar flames in mesoscale divergent channels and prediction of burning velocity at high temperatures, Combust. Sci. Technol., 185: 645-660, 2013 